\title{
Densidad y manejo de ejes en plantas injertadas de tomate indeterminadas en invernadero
}

\author{
Claudia Severino; Rafael Elizondo; Juan E Álvaro; Eduardo Oyanedel
}

Pontificia Universidad Católica de Valparaíso (PUCV), Quillota, Valparaíso, Chile; claudia.severino@hpl.cl; rafael.elizondo@hpl.cl; juaneugenio.alvaro@pucv.cl; eduardo.oyanedel@pucv.c ${ }^{1}$

\section{RESUMEN}

El uso de plantas injertadas en tomate es una práctica habitual que busca controlar enfermedades vasculares. Aun cuando las plantas injertadas son más caras, cuando se usa un portainjerto vigoroso es posible conducir en multi-ejes, usando una baja densidad de plantas por hectárea. Con el objetivo de disminuir los costos directos en mano de obra y el valor de las plantas, se evaluó densidades de $29.000,26.000,23.000$ y 20.000 ejes/ha, con conducción a 3 y 4 ejes por planta. El cultivo se realizó en un invernadero con cubierta de polietileno, trasplantando a fines de enero; se cosechó entre mayo y agosto (fines de otoño e inverno), cuantificando el rendimiento, tamaño del fruto y área foliar, analizando los datos con regresión cuadrática. El rendimiento aumentó de 81 a 103 t/ha entre la menor y la mayor densidad de ejes/ha, pero solo se presentó una diferencia de 4 t/ha entre los sistemas de conducción. Comparando los niveles extremos de densidad (29.000 y 20.000 ejes/ha), área foliar aumentó en $2 \%$, y diámetro ecuatorial del fruto aumentó de 7,4 a $7,8 \mathrm{~cm}, \mathrm{y}$ peso aumentó de 178 a $199 \mathrm{~g}$ /fruto. El sistema de conducción generó diferencias de $0.1 \mathrm{~cm}$ en el diámetro del fruto, mientras que el área foliar y el peso del fruto no fueron afectados. A pesar que la baja densidad genera una reducción del $30 \%$ en el costo de mano de obra directa, el mayor margen neto se estimó para la conducción en 3 ejes, a una densidad de 29.000 ejes/ha.

Keywords: Solanum lycopersicum, conducción, costos de producción.

\begin{abstract}
Planting density and training in grafted indeterminate tomato plants grown under plastic cover

Grafting in tomato is a regular production practice aimed to control vascular diseases. Even though grafted plants are more expensive, when a vigorous rootstock is used the plants can be trained using multiple leading shoots, reducing the number of plants per hectare. Seeking to reduce direct labor cost and the cost of the plants, this experiment tested 29,000,26,000, 23,000 and 20,000 leading shoots/ha, training the plants with either 3 or 4 leading shoots each. Plants were grown in a polyethylene-covered greenhouse, transplanting at the end of January; the harvest was done from May until August, quantifying yield, fruit size and leaf area, analyzing the data with quadratic regression. Yield increased from 81 to $103 \mathrm{t} / \mathrm{ha}$ when comparing the lowest and the highest density of shoots/ha, but the difference between the training systems was only $4 \mathrm{t} / \mathrm{ha}$. When the two extremes of the density were compared $(29,000$ vs 20,000 shoots/ha), leaf area increase by $2 \%$, the equatorial fruit diameter increased from 7.4 to $7.8 \mathrm{~cm}$, and the weight increased from 178 to $199 \mathrm{~g} /$ fruit. The training system showed difference of $0.1 \mathrm{~cm}$ in fruit diameter, while the leaf area and fruit weight were not affected. While the lowest density of shoots per hectare had a $30 \%$ reduction in direct labor cost, the largest net profit margin was observed when using 3 shoots/plant and 29,000 shoots/ha.
\end{abstract}

Keywords: Solanum lycopersicum, training, production costs.

(Recebido para publicação em 11 de julho de 2016; aceito em 28 de agosto de 2017) (Received on July 11, 2016; accepted on August 28, 2017)

$\mathrm{E}^{\mathrm{a}}$ 1 cultivo convencional de tomate (Solanum lycopersicum) en invernadero se realiza con variedades de crecimiento indeterminado sobre su propio pie, con densidades de plantación entre 24,000 a 30,000 plantas/ha. Las plantas se conducen con uno o dos ejes (tallos) por planta para maximizar el uso del espacio de la estructura de invernadero, dado su alto costo. La injertación aparece en los últimos años debido a la necesidad de eliminar el uso de bromuro de metilo en la fumigación del suelo. Los portainjertos son parte del manejo de enfermedades de suelo que afectan al cultivo de tomate, ya que cuentan con resistencia o tolerancia a algunas enfermedades como las causadas por Phytopthora, Pyrenochaeta lycopersici, Verticillium y Fusarium (Álvarez, 2012) y en algunos casos aumenta el vigor y la calidad de los frutos cosechados de la variedad comercial (Flores et al., 2010). Sin embargo, el cultivo con plantas injertadas supone un aumento de al menos cuatro veces el costo de las plantas, respecto de las plantas sobre su propio pie. Por este motivo se están utilizando sistemas de conducción de 3 y 4 ejes por planta, pero no se ha cuantificado el real impacto en la productividad. Además se debe considerar que la densidad de plantación, así como el sistema de conducción y el entutorado deben maximizar la intercepción de radiación por el cultivo, en especial en invierno cuando la menor radiación puede causar menor desarrollo de las plantas (Velasco 
et al., 2011), ya que la reducción de la radiación implica una reducción lineal del rendimiento.

La reducción en la densidad de plantación podría significar mantener el rendimiento por hectárea del cultivo; es así como en evaluaciones de densidad realizadas por Cruz et al. (2003) encontraron que densidades de 4 y 5,3 plantas $/ \mathrm{m}^{2}$ alcanzaron rendimientos similares. Por otro lado, se hace necesario encontrar la densidad adecuada a cada realidad productiva, ya que según señala un estudio conducido por Amundson et al. (2012) en tomate hidropónico, la mayor distancia de plantación $(71 \mathrm{~cm})$ produjo mayor cantidad de frutos por planta y de mayor calibre, respecto de una menor distancia de plantación $(30 \mathrm{~cm})$, no obstante la producción por unidad de superficie disminuyó. Heuvelink (1995) evaluó el efecto de la densidad de plantación sobre la distribución de biomasa hacia los frutos y encontró que frente a una alta densidad (usando 3,1 -2,1 y 1,6 plantas/ $\mathrm{m}^{2}$ ), el crecimiento de la planta se redujo significativamente; sin embargo la distribución de la materia seca no se afectó. Peil \& Gálvez (2004) en un estudio en plantas de tomates sobre su propio pie e injertadas, en sistema hidropónico, reportaron que una mayor densidad de plantas sobre su propio pie aumentó el rendimiento de la cosecha precoz, sin embargo el peso promedio del fruto se vio afectado de manera negativa; los tratamientos con plantas injertadas tuvieron efecto negativo en la precocidad y no en la cosecha total obtenida. Rahmatian et al. (2014) reportan un aumento de producción de un $27 \%$ al usar plantas injertadas en portainjerto King-Kong versus plantas injertadas en su propio pie. Además de mayor área foliar, diámetro de tallo y peso fresco y seco de las raíces.

Dada la situación de escasez de mano de obra y alto costo de las plantas injertadas, en esta investigación se busca evaluar los efectos de la densidad de tallos por unidad de superficie. Se espera responder a las siguientes preguntas: ¿Es posible mantener o mejorar los niveles de productividad y calidad al bajar la densidad? ¿Qué impacto sobre el ingreso tiene la baja densidad de ejes productivos?

\section{MATERIAL Y MÉTODOS}

El experimento se realizó en un invernadero de producción comercial cerca de la ciudad de Quillota, Chile $\left(32^{\circ} 54^{\prime} \mathrm{S}, 71^{\circ} 12^{\prime} \mathrm{W}\right.$, elevación 151 $\mathrm{m})$. El clima posee una prolongada estación seca con 300 a $400 \mathrm{~mm}$ de lluvia concentrada en invierno y una importante presencia de nubes debido a su cercanía con el mar. La temperatura media diaria durante el crecimiento de los frutos del primer al sexto racimo fue $15,0,14,4,13,5,12,9,11,9$ y $11,3^{\circ} \mathrm{C}$, respectivamente. Para los mismos estados fenológicos, la radiación media diaria fue 530, 491, 472, 432, 420 y 440 $\mathrm{W} / \mathrm{m}^{2}$. La textura de suelo es franca con un $45,3 \%$ de arena, $22,7 \%$ arcilla y $32 \%$ de limo (Nitrógeno 4,89 mg/L; Fósforo $5,62 \mathrm{mg} / \mathrm{L}$; Potasio $26,4 \mathrm{mg} / \mathrm{L} ; \mathrm{pH} 7,6$; Conductividad eléctrica $0,21 \mathrm{dS} / \mathrm{m})$. El aporte de nutrientes durante el período de cultivo fue de $340 \mathrm{~kg} / \mathrm{ha}$ de $\mathrm{N} ; 63,4$ $\mathrm{kg} / \mathrm{ha}$ de $\mathrm{P} ; 1200 \mathrm{~kg} / \mathrm{ha}$ de $\mathrm{K} ; 6,5 \mathrm{~kg} / \mathrm{ha}$ de Ca y $16,8 \mathrm{~kg} / \mathrm{ha}$ de $\mathrm{Mg}$, de acuerdo a la recomendación de Duimovic (2011).
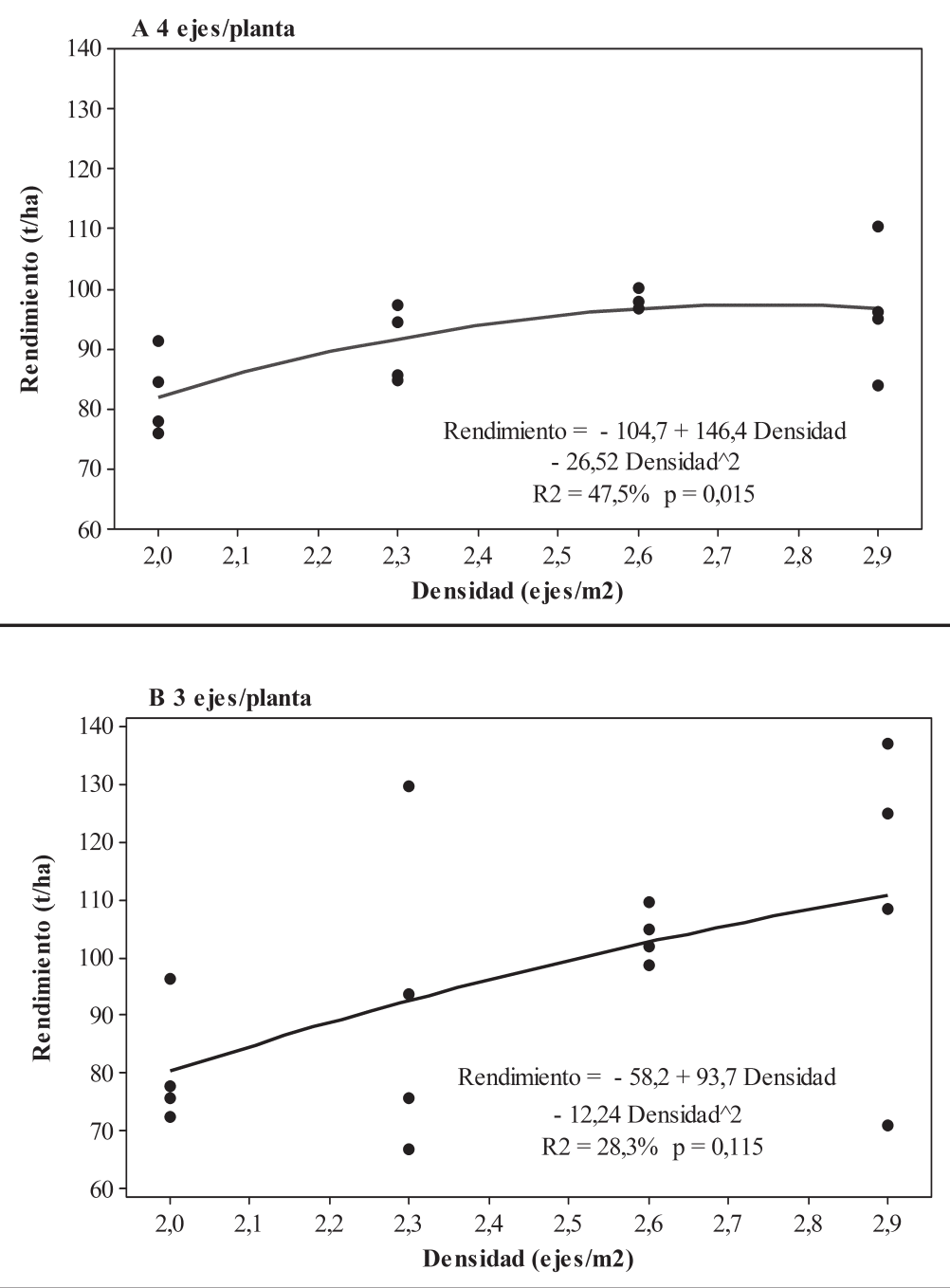

Figura 1. (A) Rendimiento comercial en función de la densidad de ejes por hectárea en plantas conducidas en 4 ejes, y (B) plantas conducidas en 3 ejes. La curva representa la ecuación de regresión cuadrática $\{(\mathrm{A})$ marketable yield as a function of the number of leading shoots per hectare for plants trained with 4 shoots, and (B) plants trained with 3 shoots. The line represents the quadratic regression equation\}. Quillota, Pontificia Universidad Católica de Valparaíso, Escuela de Agronomía, 2014. 
El experimento se realizó en 3 naves de invernadero de madera de $4,2 \mathrm{~m}$ de altura máxima al cenit, con 7,2 $\mathrm{m}$ de ancho y $34 \mathrm{~m}$ de largo. Se utilizó $2 \mathrm{~m}$ de borde al comienzo y al final de las mesas de cultivo.

La unidad experimental correspondió a una parcela de 10 metros lineales de mesa de cultivo por un metro de ancho, con 13 a 9 plantas cada una, según el tratamiento. Dos factores fueron estudiados, 4 densidades de plantación y 2 métodos de conducción ( 3 y 4 ejes/ planta). Los tratamientos fueron: 7.250 plantas/ha a 4 ejes; 6.500 plantas/ha a 4 ejes; 5.750 plantas/ha a 4 ejes; 5.000 plantas/ha a 4 ejes; 9667 plantas ha a 3 ejes; 8667 plantas/ha a 3 ejes; 7667 plantas/ha a 3 ejes; 6.667 plantas/ha a 3 ejes. Estas combinaciones resultan en densidades de 29.000, 26.000, 23.000 y 20.000 ejes/ha, tanto para la conducción en 3 como en 4 ejes. La densidad de plantación tradicional usada por el agricultor corresponde a 29.000 ejes/ ha (7.250 plantas/ha con conducción a 4 ejes).

Se utilizó el portainjerto Emperador (híbrido interespecífico, Rijk Zwaan) y Naomi (Enza Zaden) como la variedad comercial. La siembra se realizó el 15 de diciembre de 2013. Las plantas de tomate fueron injertadas por debajo de las hojas cotiledonares, el 19 de enero. El tallo principal de las plantas fue despuntado dos días antes del trasplante. Las plantas fueron trasplantadas con 4 hojas verdaderas, cuando los brotes laterales tenían menos de $1 \mathrm{~cm}$, el día 29 de enero. Los brotes laterales se desarrollaron a partir de las yemas axilares de las cuatro primeras hojas en la base de la planta. Dos brotes fueron conducidos en cada lado de la mesa de cultivo para los tratamientos a 4 ejes y en los tratamientos a 3 ejes se alternó 1 y 2 ejes a cada lado de la mesa. Los brotes laterales fueron amarrados con una cinta de polipropileno, desde su base en espiral hasta el alambre de soporte a 2 $\mathrm{m}$ de altura mientras crecían, separados entre ellos a $40 \mathrm{~cm}$. Una vez alcanzado un diámetro de fruto mayor a $1 \mathrm{~cm}$ en la región distal de cada racimo, se realizó deshoje eliminando manualmente una hoja dicho simpodio. Cada eje fue despuntado por encima de la tercera hoja sobre el séptimo racimo. La cosecha de frutos se realizó dos veces por semana, entre el 12 de mayo y el 14 de agosto, hasta completar la colecta de frutos del sexto racimo.

Se evaluó el peso individual de fruto y el diámetro ecuatorial de fruto para cada racimo. Frutos con diámetro menor a $6,4 \mathrm{~cm}$ fueron clasificados como no comerciales. El área foliar se estimó en base a la fórmula de Reis et al. (2013): $\mathrm{AF}=\mathrm{CLf}$; donde C: largo de hoja, L: ancho de hoja y f: factor 0,59 . Se midió la hoja más distal de cada simpodio, una vez terminada la expansión foliar.

Se realizó un análisis de costos e

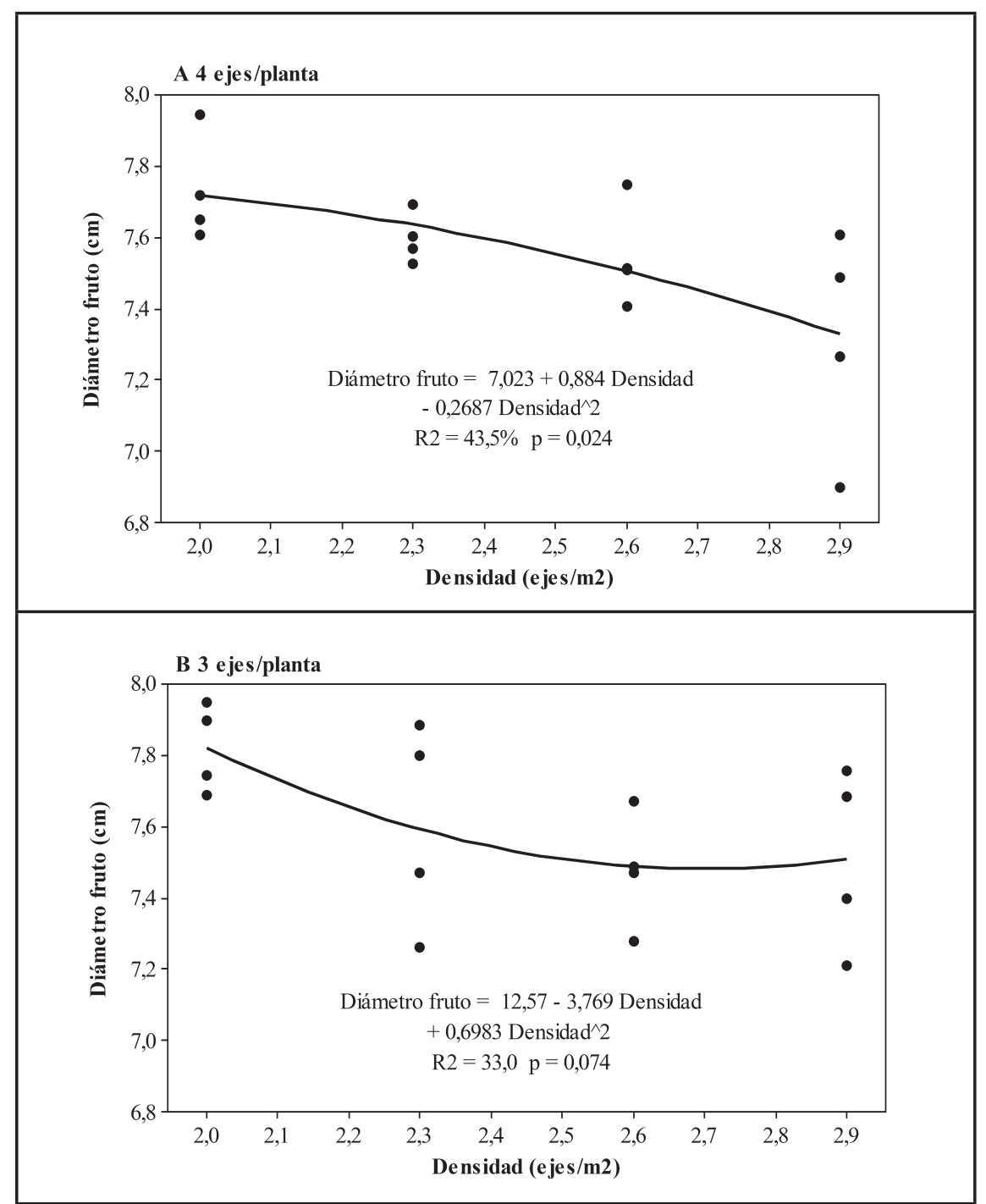

Figura 2. (A) Diámetro ecuatorial del fruto en función de la densidad de ejes por hectárea en plantas conducidas en 4 ejes, y (B) plantas conducidas en 3 ejes. La curva representa la ecuación de regresión cuadrática $\{(\mathrm{A})$ equatorial fruit diameter as a function of the number of shoots per hectare in plants trained with 4 leading shoots, and (B) plants trained with 3 leading shoots. The line represents the quadratic regression equation\}. Quillota, Pontificia Universidad Católica de Valparaíso, Escuela de Agronomía, 2014. 
En base a los ingresos y egresos, se calculó el margen neto. Los precios de la fruta fueron proporcionados por el agricultor que facilitó el invernadero para el ensayo (Sr. José Vargas Ponce), y el costo de las plantas se obtuvo en el vivero donde éstas fueron adquiridas (Vivero Agroplantines, Quillota).

Se utilizó un diseño completamente al azar con cuatro repeticiones. Las variables respuesta se analizaron a través de regresión cuadrática, utilizando el software Minitab versión 17. Se analizó por separado la respuesta del cultivo con 3 y 4 ejes por planta. Los promedios de cada repetición, para el ciclo completo de cultivo, fueron utilizados para construir los modelos cuadráticos.

\section{RESULTADOS Y DISCUSIÓN}

El rendimiento comercial fue modificado por la densidad de ejes, con una respuesta más marcada en las plantas conducidas en cuatro ejes (Figura 1). En las plantas conducidas en cuatro ejes, los valores predichos por la ecuación cuadrática son 80 t/ha para la densidad más baja y se estabilizan en 95 t/ha para las densidades medias y alta (Figura 1A). El rendimiento aumentó progresivamente al incrementar la

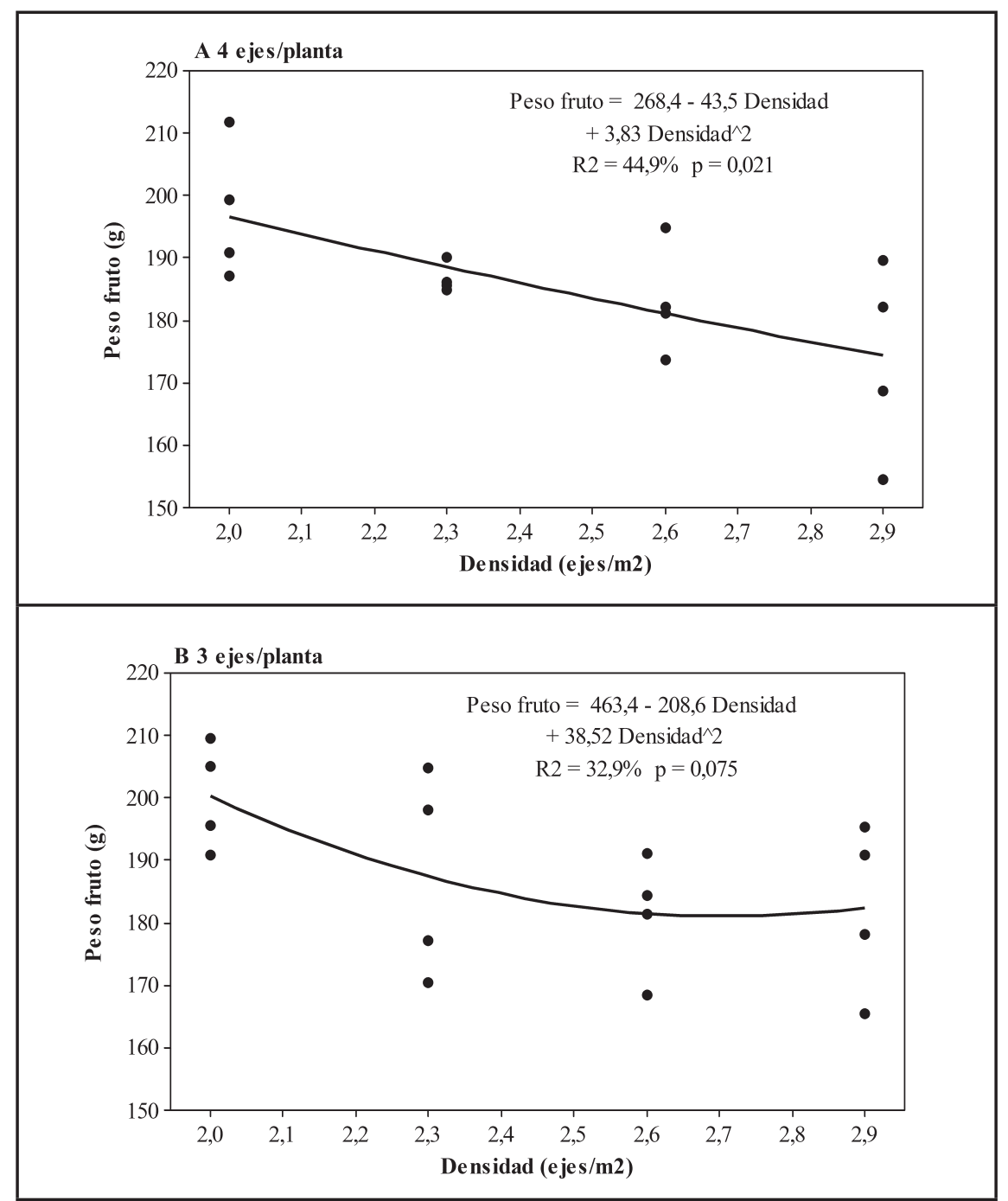

Figura 3. (A) Peso del fruto en función de la densidad de ejes por hectárea en plantas conducidas en 4 ejes, y (B) plantas conducidas en 3 ejes. La curva representa la ecuación de regresión cuadrática $\{(\mathrm{A})$ fruit weight as a function of the number of shoots per hectare in plants trained with 4 leading shoots, and (B) plants trained with 3 leading shoots. The line represents the quadratic regression equation\}. Quillota, Pontificia Universidad Católica de Valparaíso, Escuela de Agronomía, 2014. densidad, con valores entre 80 a 105 $\mathrm{t} / \mathrm{ha}$ en plantas conducidas en tres ejes (Figura 1B). Sin embargo, solo un $28,3 \%$ de la variabilidad en el rendimiento pudo ser explicada por la densidad en las plantas conducidas en 3 ejes $(p=0,115)$, mientras que el $R^{2}$ en las plantas conducidas en cuatro ejes fue $47,5 \%(\mathrm{p}=0,015)$.

Ara et al. (2007) obtuvieron un mayor rendimiento comercial con la mayor distancia de plantación (50 $\mathrm{cm})$ respecto de la menor distancia de plantación $(40 \mathrm{~cm})$, con $82,30 \mathrm{v} / \mathrm{s}$ $68,32 \mathrm{t} / \mathrm{ha}$ respectivamente; además este último produjo un menor número de frutos por planta. Un resultado similar fue obtenido por Amundson et al. (2012), quienes observaron un menor rendimiento por hectárea en las plantas distanciadas a $70 \mathrm{~cm}$ respecto de las plantadas a $30 \mathrm{~cm}$. Davis \& Estes (1993) indican que si el objetivo del agricultor es aumentar rendimiento de frutos de mayor tamaño, se debe bajar la densidad de plantación o realizar podas de ejes temprana (brotes 5 a $10 \mathrm{~cm}$ de largo). Wamser et al. (2012) observaron un aumento en la producción comercial con alta densidad (44.000 plantas/ha) respecto de la densidad tradicional (22.000 plantas/ha).

La competencia entre frutos fue más marcada en plantas conducidas en cuatro ejes, las que mostraron una mayor dependencia del diámetro del fruto en función de la densidad de ejes. El diámetro promedio del fruto se mantuvo por sobre el calibre comercial para todas las densidades, en ambos sistemas de conducción. El modelo cuadrático predice una reducción del diámetro del fruto de $7,7 \mathrm{~cm}$ hasta 7,4 $\mathrm{cm}\left(\mathrm{R}^{2}=43,5 \% ; \mathrm{p}=0,024\right)$ para plantas conducidas en cuatro ejes (Figura 2A). En las plantas conducidas en tres ejes el diámetro predicho del fruto es 7,8 $\mathrm{cm}$ para la densidad más baja, y se estabiliza en 7,5 cm para las densidades intermedias y la más alta (Figura $2 \mathrm{~B}$; $\left.\mathrm{R}^{2}=33,3 \% ; \mathrm{p}=0,074\right)$. La competencia entre frutos también se presentó en la variable peso del fruto, con una mayor dependencia de la densidad en plantas conducidas en cuatro ejes que en las plantas formadas con tres ejes. Para las plantas conducidas en cuatro ejes 


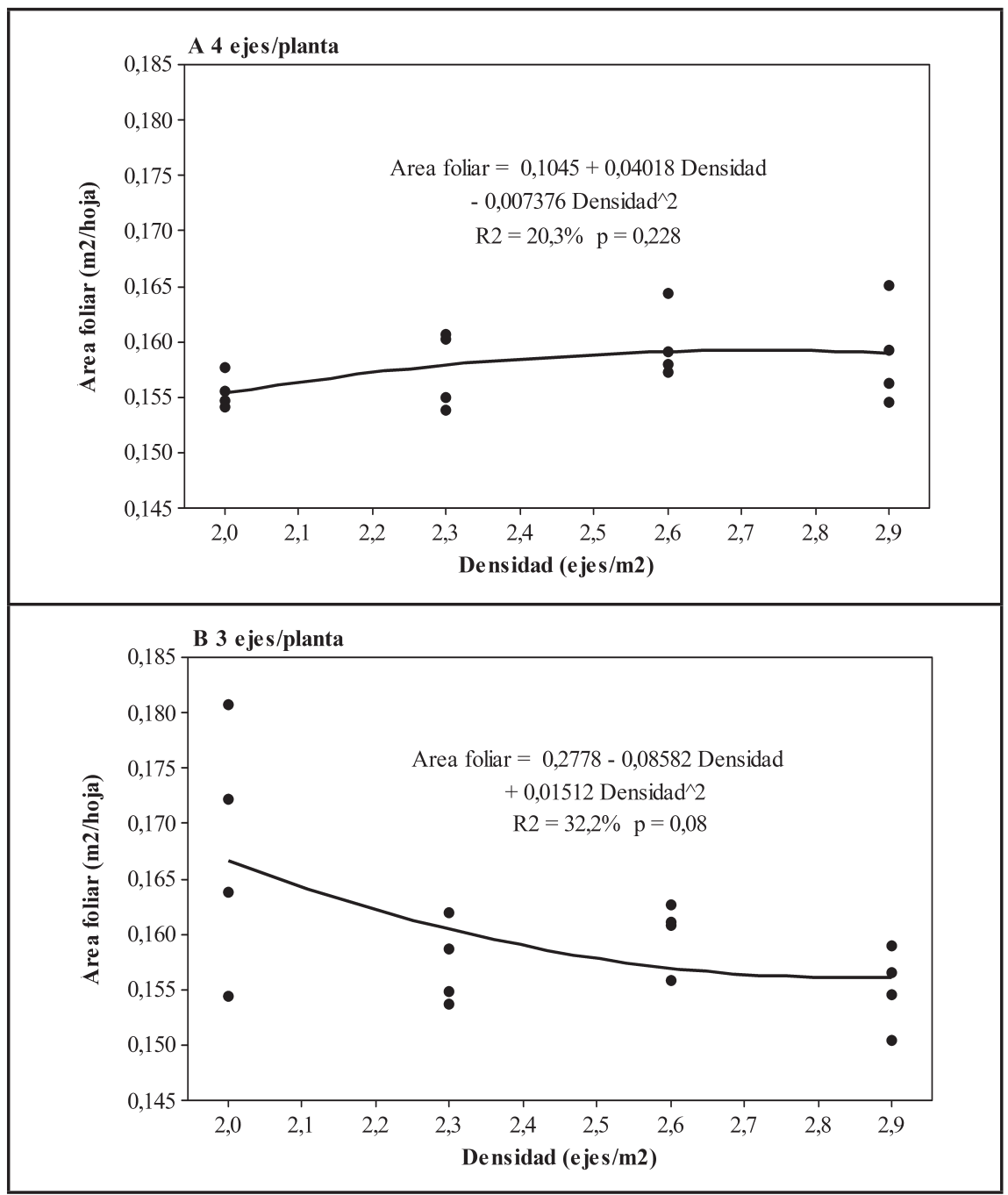

Figura 4. (A) Área foliar en función de la densidad de ejes por hectárea en plantas conducidas en 4 ejes, y (B) plantas conducidas en 3 ejes. Media corresponde a una hoja del primer al sexto simpodio. La curva representa la ecuación de regresión cuadrática $\{(\mathrm{A})$ leaf area as a function of the number of shoots per hectare in plants trained with 4 leading shoots, and (B) plants trained with 3 leading shoots. Data represent the mean of one leaf, measured from the first to the sixth sympodium. The line represents the quadratic regression equation $\}$. Quillota, Pontificia Universidad Católica de Valparaíso, Escuela de Agronomía, 2014.

el modelo cuadrático predice valores extremos de 195 y $175 \mathrm{~g}$ /fruto para baja y alta densidad, respectivamente, con $\mathrm{R}^{2}=44,9 \%(\mathrm{p}=0,021$; Figura $3 \mathrm{~A})$. En las plantas conducidas en tres ejes la respuesta es plana en las densidades medias y bajas, con un valor predicho de $185 \mathrm{~g} /$ fruto, mientras que solo en la densidad más baja el modelo predice un peso de $200 \mathrm{~g} /$ fruto (Figura $3 \mathrm{~B}$; $\mathrm{R}^{2}=$ $32,9 \% ; p=0,075)$. Considerando ambos sistemas de conducción, el máximo peso fue $199 \mathrm{~g}$ /fruto en la densidad de 20.000 ejes/ha, siendo un $10 \%$ al peso de los frutos en la densidad más alta $(29.000$ ejes/ha).
La respuesta observada fue similar a lo reportado por Qiu et al. (2013) quienes reportan que a medida que aumentó la densidad de plantación el peso promedio de los frutos disminuyó, junto con el número promedio de frutos por planta. Esto coincide con lo señalado por Azevedo et al. (2010) en donde en cultivo de tomate cherry, plantado a $0,6 \times 1,5 \mathrm{~m}$ compensó su producción respecto del plantado a $0,4 \times 1,5 \mathrm{~m}$ al alcanzar un diámetro y peso promedio de fruto mayor.

En una alta densidad es esperable una menor radiación al interior de la canopia. La época climática en que se desarrollaron estos racimos pudo haber afectado negativamente el desarrollo de los frutos que crecieron en pleno invierno; así también lo señalan Bérnard et al. (2015) en donde los frutos que cosecharon de plantas creciendo bajo sombra tuvieron un menor peso seco respecto de las desarrolladas bajo condiciones normales, con 56,8 v/s 140,2 $\mathrm{g}$ peso seco por planta respectivamente. Fan et al. (2013) trabajaron con plantas jóvenes de tomate creciendo bajo condiciones controladas de radiación y observaron un mayor peso fresco y seco de fruto, además de un mayor diámetro de tallo con mayor intensidad de luz 300 a $550 \mu \mathrm{mol} / \mathrm{m}^{2} / \mathrm{s}$, respecto a 50 a $200 \mu \mathrm{mol} / \mathrm{m}^{2} / \mathrm{s}$.

En las plantas conducidas en cuatro ejes el área foliar no se modificó significativamente con la densidad (Figura 4A; $R^{2}=20,3 \%$; $=0,228$ ). En contraste, en las plantas conducidas en tres ejes el área foliar fue mayor en la densidad más baja, y se mantuvo constante en las densidades intermedias y la más alta (Figura $4 \mathrm{~B} ; \mathrm{R}^{2}=32,2 \%$; $\mathrm{p}=$ $0,08)$. La estimación del área foliar se realizó midiendo el largo y ancho de las hojas completamente expandidas; esta medición coincide con la metodología usada por Schwarz \& Klaring (2001) y Astegiano et al. (2001) en donde el largo y ancho de las hojas entregan una buena estimación del área foliar de las plantas de tomate. Edwards et al. (2010) reportan una mayor masa foliar por unidad de área en el tercio superior de la canopia, descendiendo de manera importante hacia la parte basal (60 a $\left.20 \mathrm{~g} / \mathrm{m}^{2}\right)$. Por otro lado Bénard et al. (2015) señalan que plantas de tomate creciendo bajo sombra presentaron un área de hoja específica $\left(\mathrm{cm}^{2} / \mathrm{g}\right.$ peso seco) mayor respecto a las que crecieron en condiciones normales. Velasco et al. (2011) señalan que si la radiación fotosintéticamente activa (PAR) no es suficiente para el cultivo de tomate se producen problemas de elongación de entre nudos, follaje abundante y disminución de la producción. En el presente ensayo no se observó mayor vigor vegetativo en la fase final del cultivo, probablemente porque las hojas del tercio superior de la planta 
Tabla 1. Costos directos de mano de obra directa y plantas; y margen neto proyectado en base al rendimiento comercial por hectárea (direct costs of labor and plants; and estimated net margin based on marketable yield per hectare). Quillota, Pontificia Universidad Católica de Valparaíso, Escuela de Agronomía, 2014.

\begin{tabular}{lcccccc}
\hline $\begin{array}{l}\text { Densidad } \\
\text { (ejes/ha) }\end{array}$ & $\begin{array}{c}\text { Conducción } \\
\text { (ejes/planta) }\end{array}$ & $\begin{array}{c}\text { Costo mano de } \\
\text { obra directa } \\
\text { (US\$/ha) }\end{array}$ & $\begin{array}{c}\text { Valor de } \\
\text { las plantas } \\
\text { (US\$/ha) }\end{array}$ & $\begin{array}{c}\text { Suma costos mano de } \\
\text { Obra directa y plantas } \\
\text { (US\$/ha) }\end{array}$ & $\begin{array}{c}\text { Ingreso } \\
\text { (US\$/ha) }\end{array}$ & $\begin{array}{c}\text { Margen neto } \\
\text { (US\$/ha) }\end{array}$ \\
\hline 29.000 & 4 & $2.345,5$ & $5.038,4$ & $7.383,9$ & $39.083,0$ & $31.699,1$ \\
26.000 & 4 & $2.117,4$ & $4.517,2$ & $6.634,7$ & $39.659,0$ & $33.024,3$ \\
23.000 & 4 & $1.869,9$ & $3.996,0$ & $5.865,9$ & $36.700,9$ & $30.835,0$ \\
20.000 & 4 & $1.628,8$ & $3.474,8$ & $5.103,6$ & $33.406,6$ & $28.303,0$ \\
\hline 29.000 & 3 & $2.345,5$ & $6.718,2$ & $9.063,6$ & $44.694,0$ & $35.630,4$ \\
26.000 & 3 & $2.117,4$ & $6.023,2$ & $8.140,6$ & $42.079,1$ & $33.938,4$ \\
23.000 & 3 & $1.869,9$ & $5.328,2$ & $7.198,1$ & $37.086,6$ & $29.888,5$ \\
20.000 & 3 & $1.628,8$ & $4.633,3$ & $6.262,1$ & $32.685,9$ & $26.423,8$ \\
\hline
\end{tabular}

se desarrollaron en el período invernal, con baja temperatura y baja radiación. El sombreamiento entre plantas fue regulado en este cultivo a través de la práctica habitual de los agricultores de deshojar las plantas. Ya que el área foliar fue reducida por este manejo, es menos probable que el cultivo responda a los tratamientos de densidad.

Al analizar la diferencia entre el ingreso calculado y estos costos, se observa que el mayor margen neto estimado se logra con 29.000 ejes/ha y una conducción con 3 ejes (Tabla 1). En la zona de cultivo los costos de mano de obra directa corresponden al $40 \%$ del total de costos del cultivo y el costo de plantas al 5\% del mismo. En consecuencia, una reducción en la cantidad de ejes por unidad de superficie impacta sustancialmente sobre los costos operacionales. El alto costo de las plantas injertadas se puede compensar mejor al conducir el cultivo en el sistema de multi-ejes, especialmente cuando se utiliza un portainjerto que confiere mayor vigor a la parte aérea de la planta (Rahmatian et al., 2014).

Se puede concluir que una alta densidad es necesaria para mantener un alto rendimiento comercial. A pesar que tanto el diámetro del fruto como el peso aumentaron al bajar la densidad, el ingreso total por unidad de superficie es mayor en alta densidad. Aun cuando el costo de mano de obra directa disminuye con la baja densidad, el mayor margen neto se logra con una alta densidad.

\section{AGRADECIMIENTOS}

Los autores agradecen al agricultor Sr. José Vargas Ponce y la asistencia técnica del Sr. Gabriel Amaro L. y la Sra. Claudia Labrín R. de Hortalizas Protegidas Limitada.

\section{REFERENCIAS}

ALVAREZ, JC. 2012. Comportamiento agronómico e incidencia de enfermedades en plantas de tomate (Solanum lycopersicum L.) injertadas. Acta Agronómica 61: 117-125.

AMUNDSON, S; DEYTON, E; KOPSELL, D; HITCH, W; MOORE, A; SAMS, C. 2012. Optimizing plant density and production systems to maximize yield of greenhousegrown "Trust" tomatoes. HortTechnology 22: 44-48.

ARA, N; BASHAR, MK; BEGUM, S; KAKON, SS. 2007. Effect of spacing and stem pruning on the growth and yield of tomato. International Journal of Sustainable Crop Production 2: 35-39.

ASTEGIANO, ED; FAVARRO, JC; BOUZO, CA. 2001. Estimación del área foliar en distintos cultivares de tomate (Lycopersicon esculentum Mill.) utilizando medidas foliares lineales. Investigación Agraria. Producción y Protección Vegetales 16: 249-256.

AZEVEDO, VF; ABBOUD, ACS; CARMO, MGF. 2010. Row spacing and pruning regimes on organically grown cherry tomato. Horticultura Brasileira 28: 389-394.

BÉNARD, C; BERNILLON, S; BIAIS, B; OSORIO, S; MAUCOURT, M; BALLIAS, P; DEBORDE, C; COLOMBIÉ, S; CABASSON, C; JACOB, D; VERCAMBRE, G; GAUTIER, H; ROLIN, D; GÉNARD, M; FERNIE, A; GIBON, Y; MOING, A. 2015. Metabolomic profiling in tomato reveals diel compositional changes in fruit affected by source-sink relationships. Journal of Experimental Botany 66: 3391-3404.

CRUZ, J; JIMÉNEZ, F; RUÍZ, J; DÍAZ, G; SÁNCHEZ, P; PERALES, C; ARELLANES, A. 2003. Evaluación de densidades de siembra en tomate (Lycopersicon esculentum) en invernadero. Agronomía Mesoamericana 14: 85-88.

DAVIS, J; ESTES, E. 1993. Spacing and pruning affect growth, yield and economic returns of staked fresh-market tomatoes. Journal of the American Society for Horticultural Science 118: 719-725.

DUimoviC, A. 2011. Metodología de fertilización en tomates indeterminados, según MAFF simplificado. In: ASTORGA, R (ed). Manual de fertilización y riego en hortalizas bajo plástico. Valparaíso: INNOVACHILE CORFO - Fedefruta. p. 41-52.

EDWARDS, D; JOLLIFFE, P; EHRET, D. 2010. Canopy profiles of starch and leaf mass per area in greenhouse tomato and the relationship with leaf area and fruit growth. Scientia Horticulturae 125: 637-647.

FAN, XX; XU, ZG; LIU, XY; TANG, CM; WANG, LW; HAN, XL. 2013. Effects of light intensity on the growth and leaf development of young tomato plants grown under a combination of red and blue light. Scientia Horticulturae 153: 50-55.

FLORES, F; SÁNCHEZ-BEL, P; ESTAÑ, M; MARTINEZ-RODRIGUEZ, M; MOYANO, E; MORALES, B; CAMPOS, J; GARCÍAABELLÁN, J; EGEA, M; FERNÁNDEZGARCÍA, N; ROMOJARO, F; BOLARÍN, M. 2010. The effectiveness of grafting to improve tomato fruit quality. Scientia Horticulturae 125: 211-217.

HEUVELINK, E. 1995. Effect of plant density on biomass allocation to the fruits in tomato (Lycopersicon esculentum Mill). Scientia Horticulturae 64: 193-201.

PEIL, R; GÁLVEZ, J. 2004. Rendimiento de plantas de tomates injertadas y efecto de la densidad de tallos en el sistema hidropónico. Horticultura Brasileira 22: 265-270.

QIU, R; SONG, J; DU, T; KANG, S; TONG, L; CHEN, R; WU, L. 2013. Response of 
evapotranspiration and yield to planting density of solar greenhouse grown tomato in northwest China. Agricultural Water Management 130: 44-51.

RAHMATIAN, A; DELSHAD, M; SALEHI, R. 2014. Effect of grafting on growth, yield and fruit quality of single and double stemmed tomato plants grown hydroponically. Research Report Protected Horticulture 55: 115-119.
REIS, S; AZEVEDO, C; ALBURQUERQUE, W; JUNIOR, FS. 2013. Índice de área foliar e produtividade do tomate sob condições de ambiente protegido. Revista Brasileira de Engenharia Agricola e Ambiental 17: 386-391.

SCHWARZ, D; KLARING, H. 2001. Allometry to estimate leaf area of tomato. Journal of Plant Nutrition 24: 1291-1309.

VELASCO, E; NIETO, R; NAVARRO, E.
2011. Cultivo del tomate en hidroponía e invernadero. Madrid: Mundi-Prensa. 126p.

WAMSER, AF; MUELLER, S; SUZUKI, A; BECKER, WF; SANTOS, JP. 2012. Produtividade de híbridos de tomate submetidos ao cultivo superadensado. Horticultura Brasileira 30: 168-174. 